\title{
Etiopathogenetic factors, thyroid functions and thyroid autoimmunity in melasma patients
}

\author{
1Dermatology Clinic, Ankara Numune Education and Research Hospital, Ankara, Turkey \\ Head of the Department: Ferda Artüz \\ ${ }^{2}$ Radiology Clinic, Ankara Numune Education and Research Hospital, Ankara, Turkey \\ Head of the Department: Bülent Sakman \\ 3Department of General Surgery, Ankara University Faculty of Medicine, Ankara, Turkey \\ Head of the Department: Nezih Erverdi \\ ${ }^{4}$ Department of Biostatistics, Faculty of Medicine, Ankara University, Ankara, Turkey \\ Head of the Department: Yasemin Yavuz
}

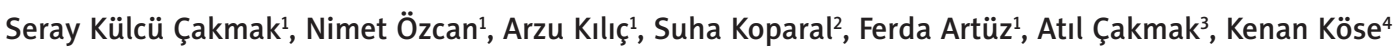

Postep Derm Alergol 2015; XXXII (5): 327-330

DOI: 10.5114/pdia.2015.54742

\begin{abstract}
Introduction: Melasma is a common chronic, acquired pigmentation disorder with a significant impact on the quality of life of patients.

Aim: To investigate the etiopathogenetic factors, thyroid functions and thyroid autoimmunity in patients with melasma.

Material and methods: Forty-five women with melasma and 45 age-matched healthy women were included in the study group. A detailed history was taken from the patients including triggering factors of melasma. Serum free triiodothyronine (FT3), free thyroxine (FT4), thyroid-stimulating hormone (TSH), anti-thyroglobulin (AbTG) and anti-thyroid peroxidase (Ab-TPO) were measured and thyroid ultrasonography was performed for each subject. Results: In $26.7 \%$ of patients, pregnancy, in $17.8 \%$, oral contraceptive use and in $13.3 \%$, intense sunlight exposure were the triggering factors. $17.8 \%$ of patients had a family history of melasma. FT4, TSH and AbTG levels were significantly higher in the patient group.

Conclusions: The results suggest that a combination of factors including pregnancy, oral contraceptive use, sunlight and genetic factors often trigger melasma. Thyroid hormones and thyroid autoimmunity may also play a role in the pathogenesis which needs to be proven by further studies.
\end{abstract}

Key words: melasma, thyroid, etiopathogenesis.

\section{Introduction}

Melasma is a localised acquired hypermelanosis which is commonly observed in women [1]. Though the exact etiopathogenesis is not clear, some factors including sun exposure, pregnancy, hormonal factors and genetic predisposition have been implicated in the pathogenesis [1-4]. Thyroid autoimmunity and thyroid diseases have also been thought to be playing a role in the pathogenesis but the number of studies on this subject is few and the results are conflicting [5-8].

\section{Aim}

The aim of this study is to investigate the etiopathogenetic factors, thyroid autoimmunity and thyroid functions in melasma patients.

\section{Material and methods}

Forty-five women with melasma aged between 24 and 49 years were included in the study group. $\mathrm{Pa}$ tients' medical history regarding thyroid diseases was taken before the initiation of the study and all of the subjects that were under treatment for known thyroid disease were excluded.

A detailed history was taken from the patients including the age of onset, duration, family history of melasma, oral contraceptive and other drug use, regular use of sunscreens, pregnancy history and associated diseases. The patients were also asked if they thought there was a relation between the onset of melasma and sun exposure, pregnancy and oral contraceptive use. Dermatological examination of the lesions were made and the distribution of the lesions were classified as malar, centrofacial

Address for correspondence: Seray Külcü Çakmak, Dermatology Clinic, Ankara Numune Education and Research Hospital, Aykon Park sit. A Blok no: 14 742, sokak Yıldızevler Çankaya, Ankara, Turkey, phone: +90 5324347169, e-mail: seraycakmak@gmail.com Received: 24.12.2014, accepted: 13.01.2015. 
or mandibular. Also the melasma area and severity in$\operatorname{dex}(\mathrm{MASI})$ were calculated and skin phototypes were determined.

Forty-five age-matched healthy women were included in the control group. The control group consisted of volunteers with no melasma lesions and who were not taking any treatment for known thyroid disease.

Serum free triiodothyronine (FT3), free thyroxine (FT4), thyroid-stimulating hormone (TSH), anti-thyroglobulin (AbTG) and anti-thyroid peroxidase (AbTPO) were measured for each subject. Thyroid ultrasonography was performed and the presence of nodules and/or inhomogeneous parenchyma of the thyroid gland were recorded.

\section{Statistical analysis}

Mann-Whitney, Pearson correlation, $\chi^{2}$, Spearman's rho and $t$-tests were used for statistical analysis and a $p$ value $\leq 0.05$ was considered statistically significant.

\section{Results}

The mean ages of the patient and control groups were $34.55 \pm 6.04$ and $34.86 \pm 5.77$ years, respectively and there was no significant difference between the ages of the patient and control groups ( $p>0.05$, t-test). The age of melasma onset was between 16 and 48 years (mean: $29.91 \pm 7.28$ years) and the duration of the disease was between 4 and 240 months (mean: $56.60 \pm 7.86$ months).

$11.1 \%$ of patients had associated diseases; 1 patient had Behçet's disease and was using colchicine, 1 had depression and was using mirtazapine, 1 had familial Mediterranean fever disease and was using colchicine, 1 had migraine and was using non-steroidal anti-inflammatory drugs and one had depression and was using alprazolam. None of the drugs were related with the onset of melasma lesions.

$17.8 \%$ of patients had a history of melasma in the first- or second-degree relatives. $68.9 \%$ of patients had a history of pregnancy and $26.7 \%$ of patients stated that their lesions started during pregnancy. $17.8 \%$ of patients stated that their lesions were related with oral contraceptive use and $13.3 \%$ of patients stated that their lesions were triggered after intense sunlight exposure. $42.2 \%$ of patients were regularly using sunscreens.

$4.4 \%$ of patients had skin phototype $2,51.1 \%$ had skin phototype 3 , and $44.4 \%$ had skin phototype 4 . The lesions were centrofacial in $42.2 \%$, malar in $42.2 \%$, centrofacial and mandibular in $11.1 \%$, malar and mandibular in $4.4 \%$ of the patients. The MASI score was between 0.4 and 9 and the mean MASI score was $4.0 \pm 2.1$.

The thyroid hormone and autoantibody levels of the patient and control groups are shown in Table 1. The FT4, TSH and AbTG levels were significantly higher in the patient group ( $p \leq 0.05)$.

No significant relation was found between MASI scores and onset age of melasma, the duration of the disease, presence of triggering factors (pregnancy related, oral contraceptive use related, intense sun-exposure related), family history of melasma, thyroid hormone and autoantibody levels (Pearson correlation, Spearman's rho test, $t$-test, $p>0.05)$.

Also no significant relation was observed between thyroid function tests and the presence of triggering factors (Mann-Whitney test, $t$-test, $p>0.05$ ).

The presence of the family history of melasma was not related with the age of onset, skin phototype, and the presence of triggering factors ( $t$ test, $\chi^{2}, p>0.05$ ).

\section{Discussion}

Melasma is a common pigmentation disorder of the skin which has a significant impact on the quality of life of patients as it frequently affects the face and is difficult to treat $[1,4]$. It occurs mostly in women of child-bearing ages with darker skin, living in areas of intense UV radiation $[2,9]$.

Melasma results from localized hyperactivity of epidermal-melanin unit leading to epidermal hyper-melanization [10]. Though the exact cause of melasma is not known, multiple factors including sunlight, pregnancy, oral contraceptive use, oestrogen and progesterone therapies, thyroid dysfunction, stress, certain cosmetics, phototoxic and anti-epileptic drugs such as carbamazepine and phenytoin are implicated in the pathogenesis [1-4].

Table 1. Thyroid hormone and autoantibody levels and thyroid ultrasonography findings in patient and control groups

\begin{tabular}{|c|c|c|c|c|}
\hline Parameter & $\begin{array}{l}\text { Patient group } \\
\text { (median } \pm S D \text { ) }\end{array}$ & $\begin{array}{l}\text { Control group } \\
\text { (median } \pm \text { SD) }\end{array}$ & Value of $p$ & Test \\
\hline FT3 $[\mathrm{pg} / \mathrm{ml}]$ & $2.98 \pm 0.45$ & $2.94 \pm 0.37$ & 0.598 & $t$-Test \\
\hline FT4 [ng/dl] & $1.16 \pm 0.19$ & $0.99 \pm 0.19$ & 0.009 & $t$-Test \\
\hline $\mathrm{TSH}[\mathrm{mlU} / \mathrm{ml}]$ & $2.36 \pm 1.24$ & $1.72 \pm 0.99$ & 0009 & $t$-Test \\
\hline abTPO [IU/ml] & $20.8 \pm 5.7$ & $30.08 \pm 10.67$ & 0.349 & Mann-Whitney test \\
\hline $\mathrm{abTG}[\mathrm{IU} / \mathrm{ml}]$ & $44.9 \pm 9.69$ & $32.04 \pm 8.23$ & 0.001 & Mann-Whitney test \\
\hline Presence of thyroid nodules, $n(\%)$ & $12(26.7)$ & $15(33.3)$ & 0.646 & $\chi^{2}$ test \\
\hline Inhomogeneous thyroid parenchyma, $n(\%)$ & $4(8.9)$ & $2(4.4)$ & 0.677 & $\chi^{2}$ test \\
\hline
\end{tabular}


The mean age of onset of melasma has been reported between 29.8 and 37.6 years previously [2, 4, 11-13]. Similarly, the age of onset was $29.91 \pm 7.28$ years in our patients. As most of the melasma patients are women and develop melasma between the second and fourth decades of life; during child-bearing ages, it supports the hormonal relationship in the pathogenesis $[1,14,15]$.

Genetic factors are also suggested in the pathogenesis as familial occurrence has been reported in $10.2-64.4 \%$ of the patients and there is a higher disease prevalence in Hispanics and Asians [2, 4, 9-13, 15]. In our study, $17.8 \%$ of the patients had a positive family history of melasma. It has been reported that subjects with a family history of melasma tended to have darker skin [13]. But we found no relation between the presence of a family history of melasma and skin phototype.

Hexsel et al. reported that patients with skin phototypes II and III and family history of melasma had earlier onset of the disorder when compared to skin phototypes IV, V and VI [2]. In our study, no relation was found between the presence of family history and the age of onset.

Ortonne et al. reported that subjects with family history of melasma appeared less likely to have the onset of melasma triggered by the use of hormonal contraception [14]. But we found no relation between the family history and presence of triggering factors.

$U V$ radiation exposure is one of the most important triggering factors in the development of melasma [2, 4, $11,15]$. UV radiation induces the increase of melanogenic activity causing the development of epidermal pigmentation [1]. Intense sun exposure has been reported as a triggering factor in $27.2 \%$ of patients in a previous study [15]. $13.3 \%$ of our patients stated that their lesions were triggered after intense sunlight exposure and $42.2 \%$ of them were regularly using sunscreens. The low incidence of intense sun exposure as a triggering factor may be due to socioeconomic and cultural factors in our country as some of our population are not exposed to intense sunlight because of their religious beliefs. The use of sunscreens should be advised as it helps to prevent melasma and increases the efficacy of other topical therapies [16].

Pregnancy is one of the triggering factors of the development of melasma, it has been found as a triggering factor in $12.1-53 \%$ of the patients, which was $26.7 \%$ in ours $[2,4,9,11,15]$. The relatively high frequency of pregnancy as a triggering factor strengthens the evidence of hormonal involvement in the pathogenesis. An increase in placental, ovarian and pituitary hormones might be the cause of pregnancy-associated melasma [13].

Ingestion of certain drugs especially oral contraceptives may trigger melasma. Oral contraceptives were reported as precipitating factors in $8.5-32.7 \%$ of the patients $[2,4,9-11,15]$. Oral contraceptive use was the triggering factor in $17.8 \%$ of our patients.

An association between thyroid disorders and melasma has been reported in some studies and it has been suggested that testing for underlying thyroid disorders can lead to improvement in certain subsets of patients $[7,12,17]$.

Lutfi et al. evaluated 84 patients with melasma. Thyroid disorders were detected in $58.3 \%$ of patients which was four-times higher than the control group. It was found that $70 \%$ of the women who developed melasma during pregnancy or while using oral contraceptives had thyroid abnormalities compared to $39.4 \%$ of patients with idiopathic melasma. They concluded that oestrogen, progesterone or both could be triggering factors in the development of melasma in women who have a particular predisposition toward both melasma and thyroid autoimmunity [7].

Kiani et al. studied 45 patients with melasma and they found that $37.8 \%$ of patients had thyroid disorders compared to $11.1 \%$ of controls and concluded that there was a significant relationship between melasma and thyroid disorders especially hypothyroidism and thyroid autoimmunity [8].

Yazdanfar and Banafsheh studied thyroid function tests and thyroid antibodies in 45 females with melasma and controls. They found that while mean serum abTPO, FT3 and TSH were higher in the patient group, the difference was not statistically significant. The number of patients with exceeding normal levels of abTPO and FT3 was significantly higher than the controls [5].

Tamega et al. found high TSH levels in $25.3 \%$ of patients with melasma and reported that high TSH levels were associated to intense sun exposure-induced melasma [15].

In our study, the FT4, TSH and abTG levels of the patient group were significantly higher and no association was found between the presence of triggering factors and thyroid hormone and autoantibody levels. Also no significant relation was found between MASI scores and thyroid hormone and autoantibody levels

The mechanism how thyroid hormones may affect melasma is not clear. Pigmentary changes may occur in thyroid diseases and hyperpigmentation has been reported to be associated with hyperthyroidism [18]. ACTH and MSH can activate melanocortin receptors in melanocytes, inducing melanogenesis [10]. It has been suggested that a strong immunoreactivity to $\alpha$-MSH on skin with melasma is one of the most important factors in the pathogenesis of the disease [19]. It has been demonstrated that the melanocortin system interacts with the hypothalamic-pituitary-thyroid axis [20].

Also an epidermal-melanin unit usually responds to certain inflammatory stimuli through melanogenesis. Melasma can be triggered or aggravated by procedures that induce skin inflammation [1, 19]. It has been suggested that thyroid hormones induce production of inflammatory cytokines. Higher circulating levels of pro-inflammatory cytokines have been shown in patients with hyperthyroidism [21]. 


\section{Conclusions}

This study confirms that multiple factors including UV exposure, oral contraceptive use, pregnancy, family history, skin type and age play a role in the development of melasma. We think that thyroid autoimmunity and thyroid hormone abnormalities might also play a role in the pathogenesis.

\section{Conflict of interest}

The authors declare no conflict of interest.

\section{References}

1. Handel AC, Miot LD, Miot HA. Melasma: a clinical and epidemiological review. An Bras Dermatol 2014; 89: 771-82.

2. Hexsel D, Lacerda DA, Cavalcante AS, et al. Epidemiology of melasma in Brazilian patients: a multicenter study. Int J Dermatol 2014; 53: 440-4.

3. Sarkar R, Arora P, Garg VK, et al. Melasma update. Indian Dermatol Online J 2014; 5: 426-35.

4. Achar A, Rathi SK. Melasma: a clinico-epidemiological study of 312 cases. Indian J Dermatol 2011; 56: 380-2.

5. Yazdanfar A, Banafsheh $\mathrm{H}$. Association of melasma with thyroid autoimmunity: a case control study. Iran J Dermatol 2010; 13: 51-3.

6. Pérez M, Sánchez JL, Aguiló F. Endocrinologic profile of patients with idiopathic melasma. J Invest Dermatol 1983; 81: 543-5.

7. Lutfi RJ, Fridmanis M, Misiunas AL, et al. Association of melasma with thyroid autoimmunity and other thyroidal abnormalities and their relationship to the origin of the melasma. J Clin Endocrinol Metab 1985; 61: 28-31.

8. Kiani A, Ahmari M, Rezvanfar MR. Association of melasma with thyroid disorders. Iran J Dermatol 2006; 9: 154-8.

9. Sardesai VR, Kolte JN, Srinivas BN. A clinical study of melasma and a comparison of the therapeutic effect of certain currently available topical modalities for its treatment. Indian J Dermatol 2013; 58: 239.

10. Handel AC, Lima PB, Tonolli VM, et al. Risk factors for facial melasma in women: a case-control study. Br J Dermatol 2014; 171: 588-94.

11. Goh CL, Dlova CN. A retrospective study on the clinical presentation and treatment outcome of melasma in a tertiary dermatological referral centre in Singapore. Singapore Med J 1999; 40: 455-8.

12. KrupaShankar DS, Somani VK, Kohli M, et al. A cross-sectional, multicentric clinico-epidemiological study of melasma in India. Dermatol Ther (Heidelb) 2014; 4: 71-81.

13. Hassan I, Kaur I, Sialy R, Dash RJ. Hormonal milieu in the maintenance of melasma in fertile women. J Dermatol 1998; 25: 510-2.

14. Ortonne JP, Arellano I, Berneburg M, et al. A global survey of the role of ultraviolet radiation and hormonal influences in the development of melasma. J Eur Acad Dermatol Venereol 2009; 23: 1254-62.

15. Tamega Ade A, Miot LD, Bonfietti C, et al. Clinical patterns and epidemiological characteristics of facial melasma in Brazilian women. J Eur Acad Dermatol Venereol 2013; 27: 151-6.

16. Sheth VM, Pandya AG. Melasma: a comprehensive update: part II. J Am Acad Dermatol 2011; 65: 699-714.
17. Sheth VM, Pandya AG. Melasma: a comprehensive update: part I. J Am Acad Dermatol 2011; 65: 689-97.

18. Mullin GE, Eastern JS. Cutaneous signs of thyroid disease. Am Fam Physician 1986; 34: 93-8.

19. Miot LD, Miot HA, Silva MG, Marques ME. Physiopathology of melasma. An Bras Dermatol 2009; 84: 623-35.

20. Martin NM, Smith KL, Bloom SR, Small CJ. Interactions between the melanocortin system and the hypothalamo-pituitary-thyroid axis. Peptides 2006; 27: 333-9.

21. Rozing MP, Westendorp RG, Maier AB, et al. Serum triiodothyronine levels and inflammatory cytokine production capacity. Age (Dordr) 2012; 34: 195-201. 